\title{
TAMANHO AMOSTRAL PARA ENSAIOS DE MECANISMOS DOSADORES DE SEMENTES DE MILHO EM ESTEIRA CARPETADA
}

\author{
VILNEI DE O. DIAS ${ }^{1}$, AIRTON DOS S. ALONÇO², DAUTO P. CARPES ${ }^{3}$, \\ ANDRÉ A. VEIT ${ }^{4}$, LUCAS B. DE SOUZA 5 .
}

\begin{abstract}
RESUMO: Devido à grande diversidade de tamanhos amostrais empregados em avaliações de desempenho de mecanismos dosadores de sementes, o objetivo deste trabalho foi determinar o tamanho de amostra para experimentos em laboratório, com dosadores de sementes tipo disco alveolado horizontal e pneumático, e estudar o efeito dos tratamentos na regularidade de distribuição de sementes da cultura do milho. Para isso, foi conduzido experimento em condições de laboratório, conforme a norma ISO 7256/1. Os tratamentos compreenderam a combinação de quatro mecanismos dosadores (dois pneumáticos e dois de disco alveolado horizontal), com três velocidades de semeadura, de 0,$7 ; 1,4$ e $2,1 \mathrm{~m} \mathrm{~s}^{-1}$. Foram utilizados os métodos de determinação do tamanho de amostra, intensidade de amostragem e bootstrap adaptado. Quanto à distribuição de sementes, os mecanismos dosadores estudados não apresentam diferenças na regularidade de distribuição, cuja regularidade caiu com aumento da velocidade de deslocamento na média dos dosadores. No que se refere ao tamanho da amostra, é possível reduzir, para a cultura do milho, para 103 espaçamentos entre sementes com nível de erro de 5\% da média amostral, independentemente da velocidade de semeadura e mecanismo dosador utilizado.
\end{abstract}

PALAVRAS-CHAVE: semeadoras de precisão, espaçamentos aceitáveis, bootstrap.

\section{SAMPLE SIZE FOR SEED MEETERS TESTS WITH CORN SEED IN CARPETED BELT}

ABSTRACT: Because to the diversity of sample sizes used in performance evaluations of seed metering mechanisms, the objective of this study was to determine the sample size for laboratory experiments with horizontal plate and pneumatic seed meters, and treatment effects in the corn seed distribution. For this, experiment was conducted under laboratory conditions, according to the international standard ISO 7256/1. The treatments comprised a combination of four metering mechanisms (two pneumatic and two horizontal plate) with three sowing speeds, 0.7, 1.4 and $2.1 \mathrm{~m}$ $\mathrm{s}^{-1}$. Methods were used for determining the sample size, sampling intensity and adapted bootstrap. Regarding the distribution of seeds, the meter mechanisms studied show no differences in the regularity of distribution, quality dropped with increasing speed. Referring to the sample size, can be reduced to 103 seed spacing with error level of 5\% of the sample mean for the corn, independent of seeding speed and metering system used.

KEYWORDS: precision seeders, acceptable index, bootstrap.

\section{INTRODUÇÃO}

O retorno econômico das culturas agrícolas anuais depende da qualidade do processo de semeadura. Pequenos descuidos nesta etapa podem gerar perdas irreversíveis no estande de plantas e, consequentemente, na produtividade (NAVID et al., 2011). Para a avaliação da regularidade de distribuição de sementes, torna-se necessária a coleta de uma amostra de espaçamentos, o que pode

\footnotetext{
${ }^{1}$ Eng ${ }^{\circ}$ Agrônomo, Prof. Doutor, Laboratório de Mecanização Agrícola do Pampa (LAMAP), UNIPAMPA/Alegrete - RS, Fone: (55) 3421-8400,vilneidias@unipampa.edu.br;

${ }^{2}$ Eng $^{\circ}$ Agrícola, Prof. Doutor, Departamento de Engenharia Agrícola, UFSM/Santa Maria - RS, alonco@ccr.ufsm.br;

${ }^{3}$ Eng $^{\circ}$ Agrônomo, Mestre em Engenharia Agrícola, Santa Maria/RS, dautocarpes@gmail.com;

${ }^{4}$ Acadêmico de Agronomia, UFSM/Santa Maria - RS, andreh_veit@hotmail.com;

${ }^{5}$ Acadêmico de Agronomia, UFSM/Santa Maria - RS, lucassouza758@yahoo.com.br.

Recebido pelo Conselho Editorial em: 16-4-2013

Aprovado pelo Conselho Editorial em: 4-5-2014
} 
ser realizado em laboratório com o auxílio de esteiras que propiciem a correta deposição das sementes, as quais, posteriormente, têm seus espaçamentos medidos, sobre os quais são estimados espaçamento médio entre sementes e índice de precisão, e também são calculados os indicadores de qualidade da regularidade de distribuição (KARAYEL, 2009). Entretanto, este tipo de aquisição de dados esbarra em diversos problemas, como o comprimento da esteira e, consequentemente, o tamanho da amostra, além do elevado tempo necessário para a tomada dos espaçamentos entre as sementes coletadas.

A norma ISO 7256/1 (ISO, 1984) estabelece os procedimentos para ensaio de semeadoras de precisão. Embora elaborada em 1983, tal norma serve até hoje como base para realização de ensaios em laboratório com semeadoras, indicando que sejam coletados 250 espaçamentos entre sementes para avaliar a regularidade de distribuição.

O conhecimento do melhor tamanho de amostra tem relevada importância para qualquer tipo de pesquisa. De acordo com MOURÃO JÚNIOR (2009), amostras grandes podem, muitas vezes, comprometer a precisão, pois quanto maior o $n$ amostral, maior a probabilidade de se encontrar diferença estatisticamente significativa entre os grupos (ou tratamentos) estudados, ainda que tal diferença não tenha definitivamente significado prático algum. Vários trabalhos tratam da determinação do tamanho ótimo de amostra em ciências agrárias, como para experimentos com a cultura do arroz irrigado (CONFALONIERI et al., 2009), estimar a população de pragas da erva-mate (LÚCIO et al., 2009), ou variáveis de experimentos envolvendo híbridos de mamoneira (CARGNELUTTI FILHO et al., 2010). Nos trabalhos conduzidos visando a avaliar a regularidade de distribuição de sementes ou plantas, tem-se usado uma ampla gama de tamanhos de amostra, variando desde 15 espaçamentos (BOZDOĠAN, 2008), 100 espaçamentos (ANANTACHAR et al., 2010), 150 espaçamentos (DIAS et al., 2009) e até 500 espaçamentos entre sementes (JASPER et al., 2006). Assim, o objetivo deste trabalho foi verificar a possível redução do tamanho de amostra para experimentos sobre esteira carpetada, com dosadores de sementes tipo disco alveolado horizontal e pneumático, para a cultura do milho, em três velocidades de deslocamento.

\section{MATERIAL E MÉTODOS}

O trabalho foi realizado sobre esteira carpetada em laboratório de acordo com a norma ISO 7256/1 (ISO, 1984). Foram utilizadas sementes de milho híbrido Dekalb ${ }^{1} 240$, peneira C2. As características dimensionais comprimento $(7,4 \pm 0,8 \mathrm{~mm})$, largura $(5,1 \pm 0,3 \mathrm{~mm})$ e espessura $(2,1 \pm 0,3 \mathrm{~mm})$ foram obtidas com paquímetro digital com resolução de $0,05 \mathrm{~mm}$ em amostras de 50 sementes, além do ângulo de repouso $\left(29,8^{\circ} \pm 3,8 \mathrm{~mm}\right)$, massa de mil sementes $(280,1 \pm 4,1 \mathrm{~g})$ e esfericidade $(58,2 \pm 5,6 \%)$ medida de acordo com a metodologia descrita por KARAYEL (2009). As sementes de milho foram depositadas sobre esteira carpetada montada em estrutura metálica tubular com 17,5 m de comprimento e $0,15 \mathrm{~m}$ de largura, a qual é forçada a tomar a forma de "V" por dois rolos verticais posicionados junto à saída do tubo condutor de sementes, de forma a reter as sementes ao tocarem na esteira. A potência necessária para movimentação da esteira e acionamento do mecanismo dosador foi fornecida por dois motores elétricos de 2,30 e 0,36 kW de potência, trifásico e monofásico, respectivamente. A adequação da rotação dos mecanismos dosadores e da velocidade de deslocamento da esteira foi realizada com o auxílio de inversores de frequência WEG $^{1}$ CFW 08 e CFW 10, respectivamente. Para fornecer a pressão negativa necessária à captação de sementes pelos mecanismos dosadores pneumáticos, foi utilizada bancada composta por um ventilador centrífugo acionado por motor elétrico trifásico, à rotação de 3.600 RPM, vacuômetro e controlador de vácuo.

Para estabelecer a velocidade de deslocamento da esteira e dos discos dosadores, foram medidos, com auxílio de tacômetro digital, valores de rotação do eixo de saída em intervalos iguais de frequência nos inversores. De posse dos pares de dados de frequência e rotação, foram traçadas equações de regressão linear entre as rotações de saída e frequências, sendo que o valor do

\footnotetext{
${ }^{1}$ A citação de marcas comerciais não implica recomendação ou não das mesmas pelo autor.
} 
coeficiente de correlação foi igual a 1. Nestas equações, foram inseridos os valores desejados de rotação, resultando nas frequências necessárias à realização do trabalho. Conhecendo-se as rotações (RPM) de entrada e saída nos pares de engrenagens, polias, eixos motrizes e discos dosadores e seus respectivos raios $(\mathrm{m})$ ou número de dentes, calcularam-se as velocidades periféricas $\left(\mathrm{m} \mathrm{s}^{-1}\right)$ das transmissões até a polia motriz da esteira ou disco do mecanismo dosador. Após a aferição da conformidade dos níveis de cada tratamento, como o proposto no trabalho, foi iniciada a coleta dos valores referentes aos espaçamentos entre sementes. A leitura da posição das sementes foi feita visualmente com auxílio de trena, com escala em milímetros, fixada ao longo de todo o comprimento da esteira, após sua passagem sob o mecanismo dosador em velocidade constante. Assim que os mecanismos dosadores, bem como a esteira, atingiram velocidade constante, estes permaneceram em funcionamento por cerca de 1 minuto para que não houvesse interferência do início do movimento de ambos. Após a completa deposição de sementes ao longo da esteira, os inversores foram desligados, fazendo com que os mecanismos cessassem o movimento, iniciandose então a tomada de espaçamentos, descontando-se as extremidades da esteira como bordadura. Devido ao fato de os dos inversores de frequência cessarem seu movimento gradativamente, minimizou-se o efeito da inércia das sementes, o que poderia causar seu rolamento ao longo da esteira carpetada.

Os tratamentos foram compostos por meio da combinação de quatro dosadores de sementes (DP1, DP2, DDH1 e DDH2), sendo dois pneumáticos e dois de disco alveolado, respectivamente, e três velocidades de deslocamento, 0,$7 ; 1,4$ e $2,1 \mathrm{~m} \mathrm{~s}^{-1}$, em delineamento de blocos ao acaso, com três repetições. As três velocidades de deslocamento foram simuladas com alteração proporcional da velocidade da esteira e da velocidade periférica dos mecanismos dosadores. Este procedimento foi adotado visando a manter o mesmo espaçamento nominal entre sementes e, consequentemente, a densidade de semeadura para ambas as culturas, simulando uma condição em que acontece elevação da velocidade de semeadura sem alteração da densidade de sementes. A densidade de semeadura foi mantida em seis sementes por metro.

Os orifícios dos dosadores de disco alveolado horizontal foram dimensionados de acordo com o ajuste do espaço livre entre orifícios e o tamanho da semente (SANTOS et al., 2003). As principais características técnicas dos mecanismos dosadores de sementes são apresentadas na Tabela 1.

TABELA 1. Principais características dos mecanismos dosadores de sementes utilizados nos ensaios. Main characteristics of the seed metering mechanisms used in the tests.

\begin{tabular}{ccccc}
\hline Parâmetro & DP1 & DP2 & DDH1 & DDH2 \\
\hline Pressão de trabalho $(\mathrm{kPa})$ & $-5,0$ & $-2,8$ & - & - \\
Diâmetro do disco $(\mathrm{mm})$ & 200 & 220 & 180 & 185 \\
Número de orifícios do disco & 24 & 30 & 28 & 28 \\
Número de fileiras & 1,0 & 1,0 & 1,0 & 1,0 \\
Diâmetro do orifício $(\mathrm{mm})$ & 5,5 & 3,6 & 8,5 & 8,5 \\
\hline
\end{tabular}

Foram utilizados dois métodos de determinação do tamanho amostral aplicados sobre as amostras-mestres de 280 espaçamentos entre sementes, com três repetições para cada tratamento. O método da intensidade de amostragem (método 1) é descrito por COCHRAN (1977), segundo a equação 1 :

$$
\mathrm{n}_{0}=\left(\mathrm{t}_{\alpha / 2^{2}} \cdot \mathrm{CV} \%^{2}\right) / \mathrm{D}^{2}
$$

em que,

$\mathrm{n}_{0}=$ tamanho da amostra;

$\mathrm{t}_{\alpha / 2}=$ valor da tabela $\mathrm{t}$, de Student, para um nível $\alpha$ de significância igual a 5\%;

$\mathrm{CV} \%=$ coeficiente de variação experimental, e 
$\mathrm{D}=$ semiamplitude do intervalo de confiança, expresso em percentual da média. Foram adotados quatro níveis de semi-amplitude: 2,$5 ; 5,0 ; 7,5$ e 10,0\% da média amostral.

A dimensão do tamanho amostral foi processada tomando a população de espaçamentos como infinita, e posteriormente fez-se a correção do tamanho amostral de acordo com a população finita (n) de 280 espaçamentos entre sementes. Esta correção ocorreu por meio da equação 2, que determina o número final de amostras (nf):

$$
\mathrm{nf}=\mathrm{n}_{0} /\left(1+\left(\mathrm{n}_{0} / \mathrm{n}\right)\right)
$$

Para a determinação do tamanho de amostra pelo método bootstrap adaptado (método 2), seguiram-se as seguintes etapas metodológicas:

Passo 1: foram estipulados cinco tamanhos de amostra: 50; 100; 150; 200 e 250 espaçamentos entre sementes para ambas as culturas; passo 2: de posse dos dados da amostra-mestre (280 espaçamentos), foram realizados, com auxílio da ferramenta "amostragem" do aplicativo Microsoft Office Excel, 100 subamostragens, com reposição de dados, para cada tamanho de amostra simulado (SILVA et al., 2011); passo 3: para cada tamanho amostral simulado, foi estimado o parâmetro percentual de espaçamentos aceitáveis das 100 subamostragens; passo 4: os resultados foram plotados em gráficos do percentual de espaçamentos aceitáveis (Y) em função do tamanho de amostra (X), e no gráfico resultante, adicionaram-se barras horizontais representativas dos limites aceitáveis e da média da amostra-mestre para facilitar a visualização e interpretação. Os limites aceitáveis foram delimitados como sendo a média da subamostra mais ou menos 2,5\%, ou seja, 2,5\% de erro admissível representando o maior nível de precisão do método da intensidade de amostragem, alinhando, assim, os resultados dos dois métodos de determinação do tamanho de amostra; passo 5: quando nenhum valor das 100 estimativas se encontrou fora do intervalo de confiança, considerou-se que a amostra representou adequadamente a população (SILVA et al., 2011).

A uniformidade de distribuição longitudinal de sementes foi avaliada de acordo com a norma ISO 7256/1 (ISO, 1984), pelo percentual de espaçamentos aceitáveis, múltiplos e falhos, com base no espaçamento nominal esperado na regulagem do mecanismo dosador (XREF). Foram classificados como falhos os espaçamentos maiores que 1,5 XREF; múltiplos, os espaçamentos menores que 0,5 XREF, e aceitáveis, os demais espaçamentos entre sementes. Os dados foram submetidos ao teste de Kolmogorov-Smirnov (SILVA \& AZEVEDO, 2009), com 5\% de probabilidade de erro, no intuito de testar a aderência dos dados à distribuição normal, e ao teste de Bartlett $(\mathrm{P}<0,01)$, para identificar a condição de homogeneidade das variâncias. Dados que não apresentaram distribuição normal de probabilidade foram transformados pela equação 3, a qual é recomendada para dados percentuais, em que $x f$ é o dado transformado, e $x$, o dado original da amostra.

$$
x f=\operatorname{arcsen} \sqrt{\frac{x}{100}}
$$

Identificadas estas condições, procedeu-se à análise das médias dos espaçamentos aceitáveis, múltiplos e falhos por meio da análise da variância $(\mathrm{P}<0,05)$. Médias diferentes significativamente, pelo teste F, foram comparadas pelo teste de Tukey, com $5 \%$ de probabilidade de erro.

\section{RESULTADOS E DISCUSSÃO}

Os dados referentes ao percentual de espaçamentos aceitáveis e falhos necessitaram de transformação para atender ao pressuposto de normalidade (Tabela 2). Com relação à homogeneidade, todas as variáveis apresentaram variâncias homogêneas depois dos dados transformados. A homogeneidade das variâncias compreende o passo fundamental para estabelecer 
um tamanho de amostra comum, e não ponderado, de acordo com as variâncias de cada parâmetro avaliado.

TABELA 2. Síntese da análise de normalidade dos dados pelo teste de Kolmogorov-Smirnov, e homogeneidade das variâncias, pelo teste de Bartlett. Summary of data normality analysis using the Kolmogorov-Smirnov test and variances homogeneity by the Bartlett test.

\begin{tabular}{|c|c|c|c|c|c|c|}
\hline \multirow[b]{2}{*}{ Variável } & \multicolumn{3}{|c|}{ Normalidade (5\%) } & \multicolumn{3}{|c|}{ Homogeneidade (1\%) } \\
\hline & Valor & Valor crítico & Normal & $\chi^{2}$ & $\chi^{2}$ crítico & Variâncias \\
\hline Aceitáveis & 0,19 & 0,15 & Não & 25,33 & 19,67 & Heterogêneas \\
\hline Aceitáveis ${ }^{1}$ & 0,14 & 0,15 & Sim & 23,53 & 24,72 & Homogêneas \\
\hline Múltiplos & 0,08 & 0,15 & Sim & 19,21 & 19,70 & Homogêneas \\
\hline Falhos & 0,19 & 0,15 & Não & 20,20 & 24,72 & Homogêneas \\
\hline Falhos $^{1}$ & 0,14 & 0,15 & Sim & 16,98 & 19,68 & Homogêneas \\
\hline
\end{tabular}

Na Tabela 3, são apresentados os desdobramentos das interações entre os fatores mecanismos dosadores e velocidade de deslocamento para as variáveis em estudo. Houve interação entre os fatores para as variáveis estudadas. A análise da variância não mostrou diferenças significativas, pelo teste $\mathrm{F}$, quanto ao fator mecanismo dosador de sementes, para as variáveis percentual de espaçamentos aceitáveis, múltiplos e falhos. Observou-se efeito apenas da velocidade de deslocamento, quando estudada a variável percentual de espaçamentos aceitáveis, exceto para o mecanismo dosador DP1. Observando-se as médias das velocidades na Tabela 3, nota-se a tendência de redução do percentual de espaçamentos aceitáveis com aumento da velocidade de semeadura na média dos tratamentos, embora este comportamento tenha-se evidenciado significativo apenas no DDH1. À exceção do DP1, os demais dosadores apresentaram algum efeito da velocidade de semeadura. Estes resultados acordam com os obtidos por MAHL et al. (2004), que pesquisando três velocidades de deslocamento $\left(1,2 ; 1,7\right.$ e $\left.2,3 \mathrm{~m} \mathrm{~s}^{-1}\right)$ e duas condições de solo, concluíram que, nas duas menores velocidades de semeadura, a eficiência na distribuição de sementes de milho foi significativamente melhor que na velocidade de $2,3 \mathrm{~m} \mathrm{~s}^{-1}$.

Quanto ao percentual de espaçamentos múltiplos, não foi observada correlação com a velocidade de semeadura, especialmente para os mecanismos dosadores pneumáticos. O percentual de espaçamentos falhos, na média das velocidades, apresentou crescimento com aumento da velocidade de semeadura.

O mecanismo dosador DP1 não sofreu efeito negativo da velocidade de semeadura, enquanto para o mecanismo DDH2, apenas a velocidade de $2,1 \mathrm{~m} \mathrm{~s}^{-1}$ reduziu significativamente o percentual de espaçamentos aceitáveis. O mecanismo dosador de sementes DDH1 apresentou diferenças significativas entre as três velocidades de semeadura, indicando sensibilidade à elevação do parâmetro para este mecanismo dosador (Tabela 3). Estes resultados corroboram os encontrados por FURLANI et al. (1999), que avaliando as velocidades de deslocamento de 0,8 e 1,4 $\mathrm{m} \mathrm{s}^{-1}$ na semeadura do milho, encontraram comportamento superior para a menor velocidade de deslocamento. 
TABELA 3. Médias das variáveis de regularidade de distribuição de sementes de milho entre os fatores mecanismos dosadores e velocidades de deslocamento. Means of the regularity distribution variables of maize seed among the mechanisms and meters speed factors.

\begin{tabular}{ccccc}
\hline & \multicolumn{4}{c}{ Velocidade de deslocamento $\left(\mathrm{m} \mathrm{s}^{-1}\right)$} \\
\cline { 2 - 4 } Dosador & 0,7 & \multicolumn{4}{c}{1,4} & 2,1 & Média \\
DP1 & & Espaçamentos aceitáveis $(\%)$ & $81,0^{\text {ns }}$ \\
DP2 & $78,0 \mathrm{aA}$ & $83,2 \mathrm{aA}$ & $81,8 \mathrm{aA}$ & 81,0 \\
DDH1 & $87,3 \mathrm{aA}$ & $79,8 \mathrm{aAB}$ & $75,9 \mathrm{aB}$ & 66,9 \\
DDH2 & $84,2 \mathrm{aA}$ & $66,2 \mathrm{aB}$ & $51,4 \mathrm{aC}$ & 87,4 \\
Média & $93,6 \mathrm{aA}$ & $89,4 \mathrm{aA}$ & $79,3 \mathrm{aB}$ & \\
& $85,8 \mathrm{~A}$ & $79,7 \mathrm{~B}$ & $72,1 \mathrm{C}$ & $11,2^{\text {ns }}$ \\
DP1 & & Espaçamentos múltiplos $(\%)$ & 8,9 \\
DP2 & $13,0 \mathrm{aA}$ & $10,5 \mathrm{aA}$ & $10,1 \mathrm{aA}$ & 13,2 \\
DDH1 & $6,5 \mathrm{aA}$ & $9,8 \mathrm{aA}$ & $10,5 \mathrm{aA}$ & 5,7 \\
DDH2 & $8,1 \mathrm{aB}$ & $15,7 \mathrm{aA}$ & $15,9 \mathrm{aA}$ & \\
Média & $3,3 \mathrm{aB}$ & $5,6 \mathrm{aAB}$ & $8,3 \mathrm{aA}$ & $11,2 \mathrm{~A}$ \\
& $7,7 \mathrm{~B}$ & $10,4 \mathrm{~A}$ & $7,8^{\mathrm{ns}}$ \\
DP1 & & Espaçamentos falhos $(\%)$ & 10,1 \\
DP2 & $9,0 \mathrm{aA}$ & $6,3 \mathrm{aA}$ & $8,1 \mathrm{bA}$ & 19,9 \\
DDH1 & $6,2 \mathrm{aB}$ & $10,4 \mathrm{aAB}$ & $13,6 \mathrm{bA}$ & 6,8 \\
DDH2 & $7,7 \mathrm{aC}$ & $18,1 \mathrm{aB}$ & $32,6 \mathrm{aA}$ & $12,4 \mathrm{bA}$ \\
Média & $3,1 \mathrm{aB}$ & $5,0 \mathrm{aB}$ & $16,6 \mathrm{~A}$ & \\
\hline
\end{tabular}

DP1: dosador pneumático 1; DP2: dosador pneumático 2; DDH1: dosador de disco alveolado 1; DDH2: dosador de disco alveolado 2; médias seguidas de mesma letra, minúscula na coluna e maiúscula na linha, não diferem pelo teste de Tukey, com 5\% de probabilidade de erro; ns: não significativo. DMS das interações: aceitáveis: 8,2 para linhas e 31,8 para colunas; múltiplos: 4,9 para linhas e 15,5 para colunas; falhos: 14,2 para linhas e 4,3 para colunas.

Alusivo ao tamanho de amostra determinado pelo método da intensidade de amostragem, a análise da variância mostrou efeito significativo apenas da semiamplitude, indicando que poderiam ser utilizados os mesmos tamanhos de amostra, independentemente do mecanismo dosador ou velocidade de semeadura, escolhendo-se apenas o nível de erro desejado $(2,5 ; 5,0 ; 7,5$ ou $10 \%)$. Por este método, não foi encontrada tendência de aumento do tamanho de amostra com o aumento da velocidade de semeadura. A análise da variância não mostrou interação entre os fatores velocidade de deslocamento e semiamplitude. Os tamanhos de amostra obtidos variaram de 12 espaçamentos entre sementes para o dosador DDH2, a $1,4 \mathrm{~m} \mathrm{~s}^{-1}$ com erro de $10 \%$ da média amostral, até 195 espaçamentos entre sementes para o mecanismo dosador DDH1, na mesma velocidade, com erro de 2,5\% da média amostral (Tabela 4). Mesmo o maior tamanho de amostra estimado pelo método da intensidade de amostragem é $22 \%$ menor que o recomendado pela ISO 7256/1 (ISO, 1984). Se observarmos a média das semiamplitudes, pode-se reduzir o tamanho de amostra em $71 \%$, com 5\% de erro da média amostral. 
TABELA 4. Tamanho de amostra mínimo para avaliação da regularidade de distribuição de sementes pelo método da intensidade de amostragem. Minimum sample size to evaluate the regularity of seed distribution determined by the sampling rate method.

\begin{tabular}{|c|c|c|c|c|}
\hline \multirow{3}{*}{$\begin{array}{c}\text { Velocidade } \\
\left(\mathrm{m} \mathrm{s}^{-1}\right)\end{array}$} & \multicolumn{4}{|c|}{ Semiamplitude (D\%) } \\
\hline & \multicolumn{4}{|c|}{ DP1 } \\
\hline & 2,5 & 5,0 & 7,5 & 10,0 \\
\hline \multicolumn{5}{|c|}{ Tamanho de amostra ( $\mathrm{n}$ espaçamentos entre sementes) } \\
\hline 0,7 & 176 & 84 & 45 & 28 \\
\hline 1,4 & 156 & 67 & 34 & 20 \\
\hline 2,1 & 155 & 67 & 35 & 21 \\
\hline \multicolumn{5}{|c|}{ DP2 } \\
\hline 0,7 & 108 & 44 & 23 & 13 \\
\hline 1,4 & 144 & 66 & 36 & 22 \\
\hline 2,1 & 165 & 81 & 45 & 28 \\
\hline \multicolumn{5}{|c|}{ DDH1 } \\
\hline 0,7 & 169 & 78 & 41 & 25 \\
\hline 1,4 & 195 & 103 & 57 & 36 \\
\hline 2,1 & 193 & 100 & 56 & 34 \\
\hline \multicolumn{5}{|c|}{ DDH2 } \\
\hline 0,7 & 130 & 55 & 29 & 17 \\
\hline 1,4 & 106 & 42 & 21 & 12 \\
\hline 2,1 & 147 & 79 & 46 & 29 \\
\hline Média & $156 \mathrm{~A}$ & $72 \mathrm{~B}$ & $39 \mathrm{C}$ & $23 \mathrm{D}$ \\
\hline
\end{tabular}

Nas Figuras 1 e 2, são apresentadas as dispersões das 100 estimativas para cada um dos seis tamanhos de amostra simulados pelo método bootstrap adaptado. Apenas dois tratamentos apresentaram valores fora dos limites aceitáveis, ambos para os dosadores de disco alveolado na velocidade de deslocamento de $2,1 \mathrm{~m} \mathrm{~s}^{-1}$. Para os tratamentos compostos pelos mecanismos dosadores pneumáticos em combinação com as três velocidades, todas as estimativas ficaram dentro do intervalo estabelecido pelos limites superior e inferior aceitáveis, indicando que poderiam ter sido utilizadas amostras menores que a de 280 espaçamentos entre sementes, inclusive a de 50 espaçamentos, para estimar o percentual de espaçamentos aceitáveis com o erro de $2,5 \%$ da média da amostra-mestre.

Para os tratamentos compostos pelos mecanismos dosadores DDH1 e DDH2, na velocidade de $2,1 \mathrm{~m} \mathrm{~s}^{-1}$, as estimativas ficaram fora do intervalo de confiança para o tamanho de amostra de 100 espaçamentos entre sementes, sugerindo a não adequação do uso de amostras menores que estas, por este método de determinação. Pode-se observar, na Figura 2, que a dispersão das estimativas são menores em relação à média, o que é evidenciado pela menor altura das barras formadas pelas plotagens de cada tamanho de amostra, ficando estas mais distantes dos limites aceitáveis. Em uma percepção inicial, o método bootstrap atribuiu menores tamanhos de amostra para os tratamentos que apresentaram maior regularidade de distribuição de sementes de milho.

Os tratamentos formados pelos mecanismos dosadores pneumáticos não apresentaram diferenças nos tamanhos de amostra simulados, independentemente da velocidade de deslocamento utilizada. Estes resultados reafirmam as conclusões de BEDIN et al. (2009) e DIAS et al. (2010), que não verificaram efeito do tamanho de amostra nas variáveis de regularidade de distribuição de sementes de uma semeadora, em experimentos conduzidos sobre leito de areia. Os autores compararam diferentes padrões amostrais, de 50 até 300 espaçamentos, e não verificaram efeito nos 
percentuais de aceitáveis, múltiplos e falhos. Desta forma, fica evidenciado o potencial para a redução do tamanho amostral nestas avaliações, independentemente da superfície ser areia ou esteira.

DP1 $x 0,7 \mathrm{~m} \mathrm{~s}^{-1}$

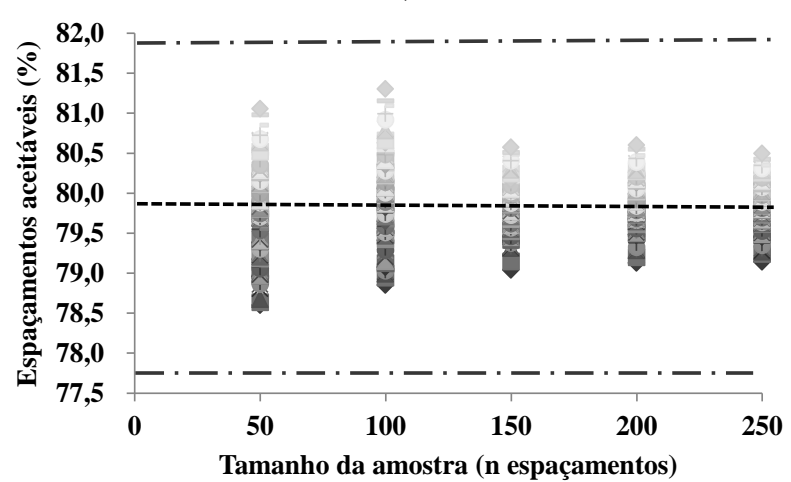

DP1 x $2,1 \mathrm{~m} \mathrm{~s}^{-1}$

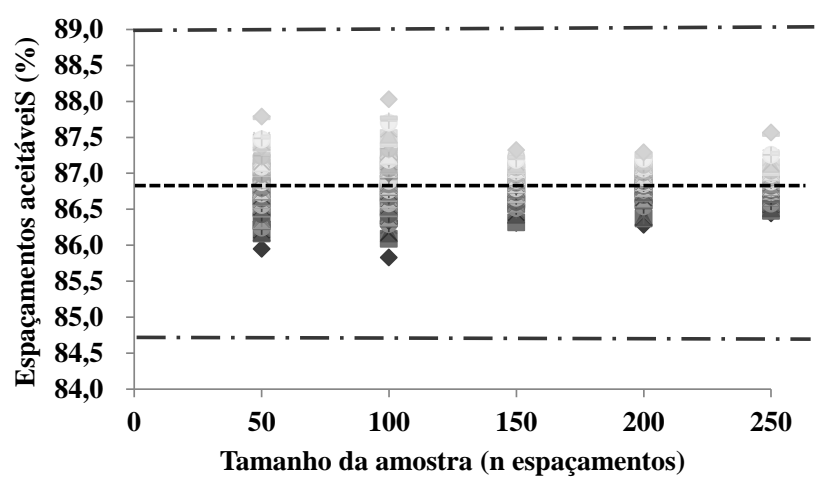

DP2 x $1,4 \mathrm{~m} \mathrm{~s}^{-1}$

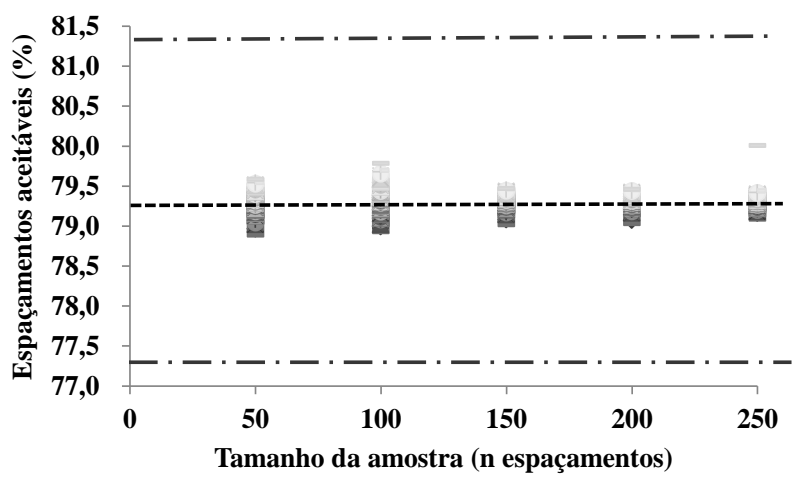

DP1 x $1,4 \mathrm{~m} \mathrm{~s}^{-1}$

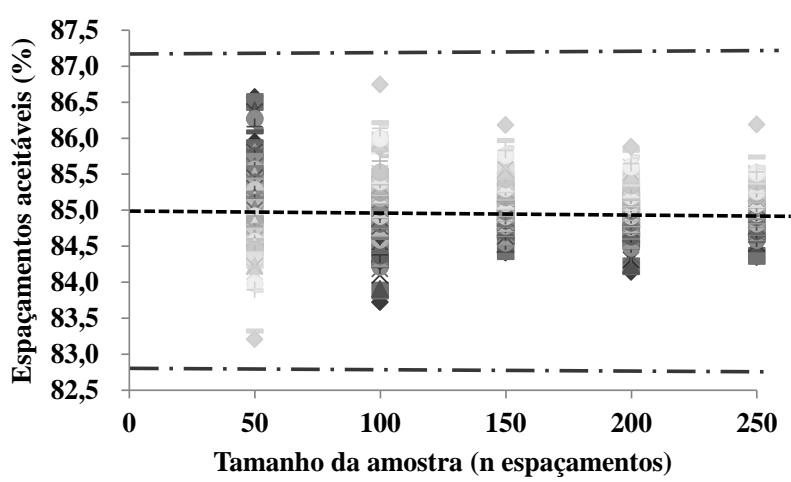

DP2 $\times 0,7 \mathrm{~m} \mathrm{~s}^{-1}$

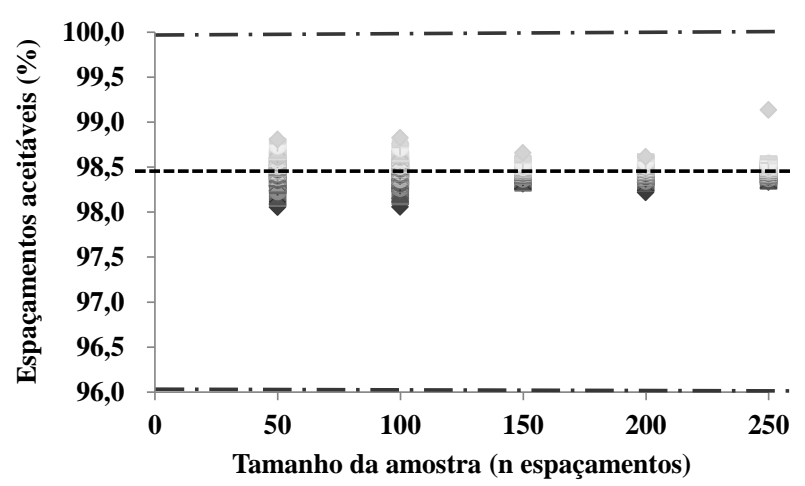

DP2 $\times 2,1 \mathrm{~m} \mathrm{~s}^{-1}$

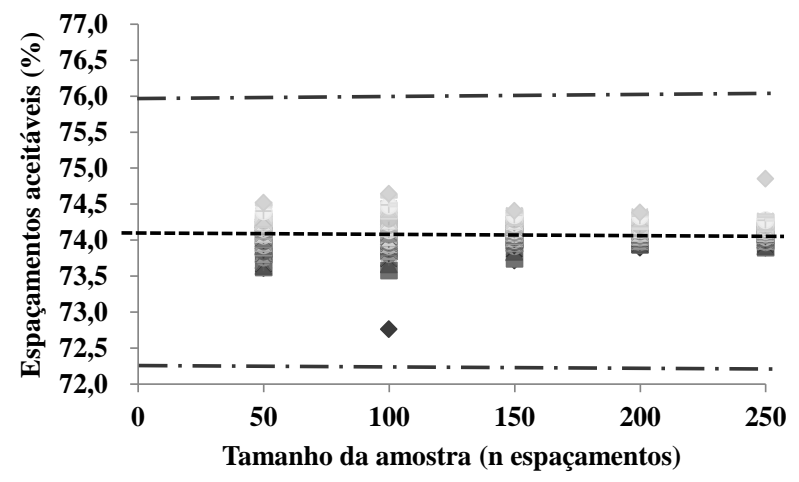

FIGURA 1. Dispersão das 100 estimativas do percentual de espaçamentos aceitáveis em função de cinco tamanhos de amostra simulados (n) para os dosadores pneumáticos; a linha tracejada indica a média da amostra-mestre; linhas-traço e ponto indicam os limites aceitáveis superior e inferior para a aceitação do tamanho de amostra. Dispersion of 100 estimates in the percentage of acceptable spacing due to 5 sizes of simulated sample (n) for pneumatic meters, the dashed line indicates the sample average; dash and point lines indicate the upper and lower acceptable limits for sample size acceptance. 

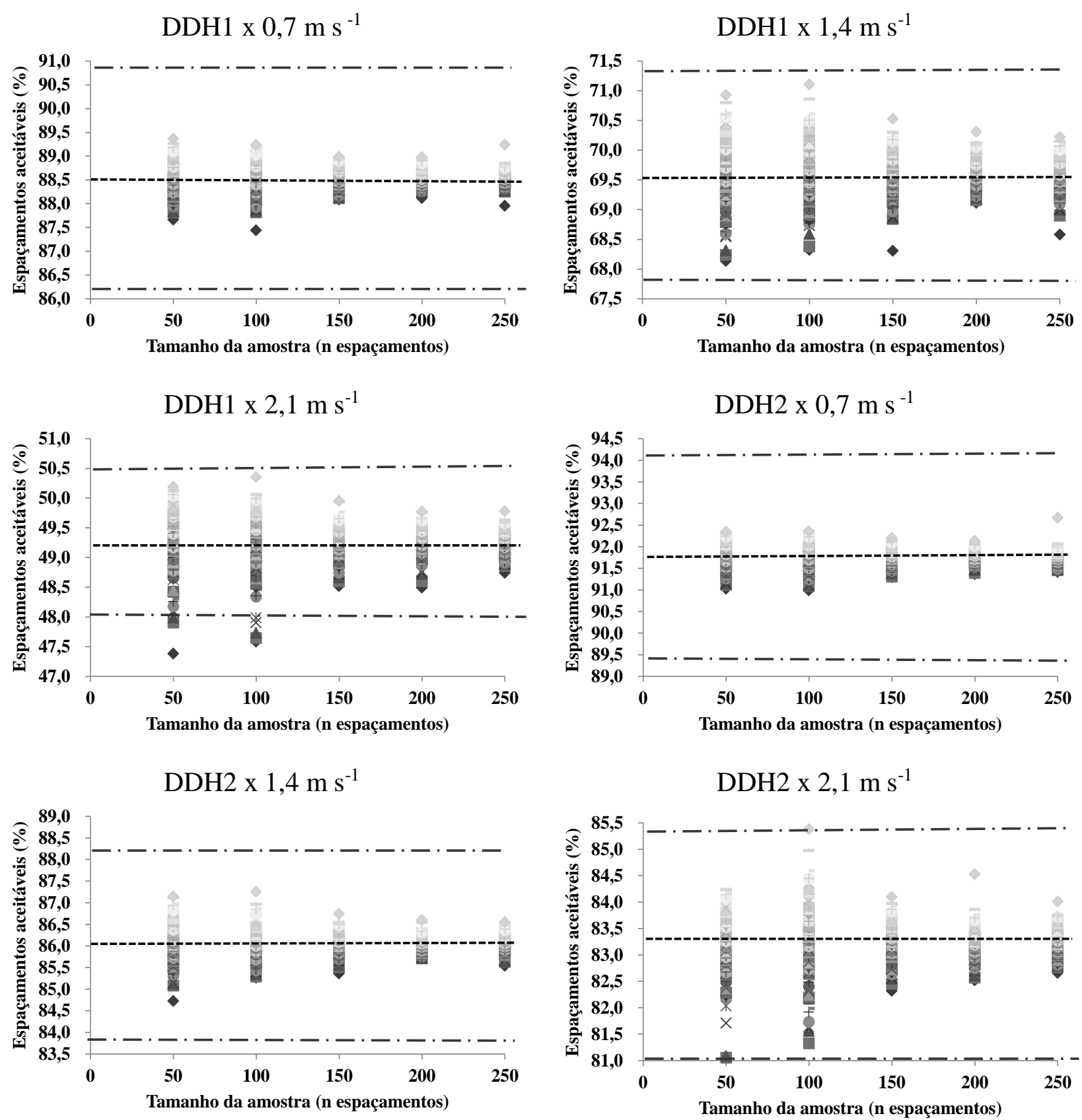

FIGURA 2. Dispersão das 100 estimativas do percentual de espaçamentos aceitáveis em função de cinco tamanhos de amostra simulados (n) para os dosadores de disco alveolado; a linha tracejada indica a média da amostra-mestre; linhas-traço e ponto indicam os limites aceitáveis superior e inferior para a aceitação do tamanho de amostra. Dispersion of 100 estimates in the percentage of acceptable spacing due to 5 sizes of simulated sample (n) for pneumatic meters, the dashed line indicates the sample average; dash and point lines indicate the upper and lower acceptable limits for sample size acceptance.

\section{CONCLUSÕES}

É possível reduzir o tamanho de amostra para avaliar a regularidade de distribuição de sementes de milho em esteira carpetada, independentemente da velocidade de deslocamento testada e do tipo de mecanismo dosador, pneumático ou de disco alveolado. Adotando-se o menor nível de erro do método da intensidade de amostragem, podem-se utilizar amostras de 103 espaçamentos entre sementes, reduzindo em 59\% o tamanho de amostra recomendado pela ISO 6256/1. Podem 
ser utilizados, em média, 23 espaçamentos entre sementes para avaliações que não necessitem de precisão experimental maior que $90 \%$, isto é, com um erro conhecido de $10 \%$.

\section{REFERÊNCIAS}

ANANTACHAR, M., PRASSANA, G.V.K.; GURUSWAMY, T. Neural network prediction of performance parameters of an inclined plate seed metering device and its reverse mapping for the determination of optimum design and operational parameters. Computers and Electronics in Agriculture, New York, v. 72, n. 2, p. 87-98, 2010.

BEDIN, P. R.; ALONÇO, A. dos S.; CARDINAL, K.; LASSEN, P.G.; DIAS, V. de O. Efeito do tamanho da amostra na distribuição de espaçamentos entre sementes por um dosador de disco alveolado. In: JORNADA ACADÊMICA INTEGRADA, 24, 2009, Santa Maria. Anais... Santa Maria: UFSM, 2009. CD-ROM.

BOZDOĠAN, A. M. Seeding uniformity for vacuum precision seeders. Scientia Agrícola, Piracicaba, v. 65, n. 3, p. 318-322, 2008.

CARGNELUTTI FILHO, A.; LOPES, S. J.; BRUM, B. SILVEIRA, T. R. da; TOEBE, M.; STORCK, L. Tamanho de amostra de caracteres em híbridos de mamoneira. Ciência Rural, San v. ta Maria, 40, n. 2, p. 280-287, 2010.

COCHRAN, W. G. Sampling techniques. 3 ed. New York: John Wiley \& Sons, 1977. 428p.

CONFALONIERI, R.; PEREGO, A.; CHIODINI, M. E.; SCAGLIA, B.; ROSENMUND, A. S.; ACUTIS, M. Analysis of sample size for variables related to plant, soil, and soil microbial respiration in a paddy rice field. Field Crops Research, Amsterdam, n. 113, p. 125-130, 2009.

DIAS, V. de O.; ALONÇO, A. dos S.; BAUMHARDT, U. B.; BONOTTO, G. J. Distribuição de sementes de milho e soja em função da velocidade e densidade de semeadura. Ciência Rural, Santa Maria, v. 39, n. 6, p. 1721-1728, 2009.

DIAS, V. de O.; ALONÇO, A. dos S.; BEDIN, P. R.; SILVEIRA, H. A. T. Estimativa do tamanho de amostra para avaliar a distribuição de sementes de milho e soja em leito de areia por uma semeadora de precisão. In: CONGRESSO BRASILEIRO DE ENGENHARIA AGRÍCOLA. 39, 2010, Vitória, Anais... Jaboticabal: SBEA, 2010, CD-ROM.

FURLANI, C. E. A.; LOPES, A.; ABRAHÃO, F. Z.; LEITE, M. A. S. Características da cultura do milho (Zea mays L.) em função do tipo de preparo do solo e da velocidade de semeadura.

Engenharia Agrícola, Jaboticabal, v. 19, n. 2, p. 177-186, 1999.

ISSO - Internacional Organization for Estandardization. ISO 7256/1: Sowing equipment - Methods of test-Part 1: Single seed drills (precision drills). 1984. 16p.

JASPER, R.; JANSZEN, U.; JASPER, M.; GARCIA, L. C. Distribuição longitudinal e germinação de sementes de milho com emprego de tratamento fitossanitário e grafite. Engenharia Agrícola, Jaboticabal, v. 26, n. 1, p. 292-299, 2006.

KARAYEL, D. Performance of a modified precision vacuum seeder for no-till sowing of maize and soybean. Soil Tillage Research, Amsterdam, v.140, n.1, p. 121-125, 2009.

LÚCIO, A. D.; NETO, J. V.; CHIARADIA, L. A.; STORCK, L. Distribuição espacial e tamanho de amostra para o ácaro-do-bronzeado da erva-mate. Revista Árvore, Viçosa, v. 33, n. 1, p. 143-150, 2009.

MAHL. D. GAMERO, C. A.; BENEZ, S. H.; FURLANI, C. E. A.; SILVA, A. R. B. Demanda energética e eficiência da distribuição de sementes milho sob variação de velocidade e condição de solo; Engenharia Agrícola, v. 24, n. 1,Jaboticabal, p.150-157. 2004.

MOURÃO JR, C. A. Questões em bioestatística: o tamanho da amostra. Revista Interdisciplinar de Estudos Experimentais, Juiz de Fora, v. 1, n. 1, p. 26 - 28, 2009. 
NAVID, H.; EBRAHIMIAN, S.; GASSEMZADEH, H. R.; MOUSAVI NIA, M. J. Laboratory evaluation of seed metering device using image processing method. Australian Journal of Agricultural Engineering, v. 2, n. 1, p. 01-04, 2011.

SANTOS, S. R. dos; PEREIRA, J. O.; WEIRICH NETO, P. H.; FEY, E. Espaço livre entre orifícios de discos dosadores e sementes de milho na eficiência de semeadura. Engenharia Agrícola, Jaboticabal, v. 24, n. 1, p. 150-157, 2003.

SILVA, A.R. da; RÊGO, E.R. do; CECON, P.R. Tamanho de amostra para caracterização morfológica de frutos de pimenteira. Horticultura Brasileira, Brasília, v. 29, n. 1, p. 125-129, 2011.

SILVA, F. de A. S.; AZEVEDO, C. A. V. de. Principal Components Analysis in the Software Assistat-Statistical Attendance. In: WORLD CONGRESS ON COMPUTERS IN AGRICULTURE, 7, 2009, Reno. Anais... Reno: American Society of Agricultural and Biological Engineers, 2009. 